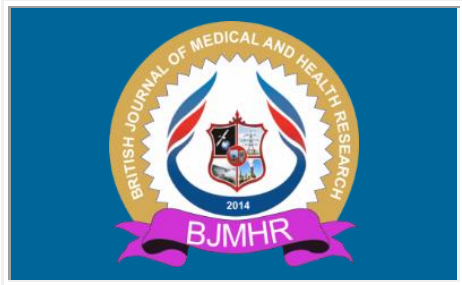

\title{
BJMHR
}

British Journal of Medical and Health Research

Journal home page: www.bjmhr.com

\section{To Elucidate Precipitating Factors, Clinical Profile and Outcome of Acute-on-Chronic Liver Failure in Cirrhotic Patients.}

\author{
Netaji Garad ${ }^{1 *}$ \\ Department of Gastroenterology \& Hepatology, Shri Balaji Institute of Medical Science, \\ Raipur, Chhattisgarh 492001.
}

\section{ABSTRACT}

An acute deterioration of liver function in a patient with previously compensated chronic liver disease is a result of relentless progression of underlying liver disease. This research was conducted to elucidate clinical profile, precipitating factors, prognostic factors, predicting the survival and outcome of Acute-on-chronic liver failure in cirrhotic patients. This prospective observational study of hundred patients was conducted in PVS Memorial Hospital, Cochin, Kerala, India in 2015-16. Patients were followed up during hospital stay and outcome was noted at 28 day. The clinical manifestations, laboratory parameters and other imaging findings were analyzed. Out of 100 patients studied, 87 were males and 17 females with sex ratio 4.9:1. The mean age of all subjects was 54.21 \pm 11.23 years. Alcohol was the primary etiology for cirrhosis in 69 (69\%) patients while $25(25 \%)$ patients etiology could be identified and labeled as NASH/Cryptogenic. 13\% had grade 3-4 hepatic encephalopathy. Liver, renal, coagulation, cerebral, circulatory and respiratory failure were seen in $67 \%, 32 \%$, $39 \%, 15 \%, 39 \%, 30 \%$, and 13\% respectively. Most frequent complication was coagulopathy i.e. INR $>2.5$, which was found in $36 \%$ of patients. The most striking differences were seen in MELD, MELD Na, APACHE II and SOFA scores between no ACLF and ACLF group. Among all four scores, APACHE II had highest sensitivity of $92.7 \%$ ( $<<0.001)$. Mortality at 28 days was recorded in 55\% (55 patients). Mortality was highest in ACLF group II (77.8\%) and ACLF group III was (95.7\%). It is evident that alcohol is the most common etiology for cirrhosis followed by NASH. APACHE II has a better sensitivity in predicting the mortality compare to other scores. More refine and precise ACLF classification system is required in order to diagnose high-risk patients and predict mortality and survival rates.

Keywords: Chronic hepatic decompensation, Cirrhosis, CLIF - SOFA score, ACLF, APACHE II, MELD, NASH.

*Corresponding Author Email: drnetajigarad@gmail.com

Received 05 March 2020, Accepted 22 March 2020

Please cite this article as: Garad N., To Elucidate Precipitating Factors, Clinical Profile and Outcome of Acute-on-Chronic Liver Failure in Cirrhotic Patients.. British Journal of Medical and Health Research 2020. 


\section{INTRODUCTION}

Liver failure can occur in a setting of normal liver (known as acute liver failure) or on a background of chronic liver damage (acute-on-chronic liver failure or ACLF). It has been well known that in a patient with previously stable chronic liver disease, occurrence of an acute insult results in rapid deterioration of liver function and subsequent decompensation. The precipitating event can vary from acute alcohol abuse, viral hepatitis (A, B or E), variceal bleeding, sepsis, adverse drug reaction or surgery. Many of these patients develop multi-organ failure, which itself signals high immediate and short term mortality. ${ }^{1}$. Acute-on-chronic liver failure (ACLF) is a relatively new term, the concept of which was first laid down in 2000 by Jalan and Williams. Patients with ACLF behave differently from decompensated liver disease. In a study by Jalan et al, immediate short term mortality in this group of patients was around $58 \%$, which suggests that there is an element of reversibility in such deterioration and it can be reversed if the patient receives prompt and adequate intensive care. ${ }^{2}$

There have been various reports and definitions of what exactly consists of ACLF. There is no consistent definition of ACLF in the literature. Each study done previously on ACLF has used its own definition, and there is no unanimity in these definitions in terms of criteria for liver failure, the acute event precipitating ACLF, and the diagnosis of underlying chronic liver disease. The consensus definition is "ACLF is an acute hepatic insult manifesting as jaundice (serum bilirubin $\geq 5 \mathrm{mg} / \mathrm{dL}$ ( $85 \mathrm{micromol} / \mathrm{L}$ ) and coagulopathy (INR $\geq 1.5$ or prothrombin activity $<40 \%$ ) complicated within 4 weeks by clinical ascites and/or encephalopathy in a patient with previously diagnosed or undiagnosed chronic liver disease/cirrhosis, and is associated with a high 28-day mortality." "3 Being more of a concept at this stage, there have been efforts to adequately characterize this group, to determine prognosticating markers and to improve survival. It has been always argued that ACLF of west differs from ACLF described in the east. Starting with the primary etiology, whereas hepatitis B virus forms a significant cause of cirrhosis in the Asia-Pacific region, those in the west have alcohol and increasingly NASH as a significant cause of cirrhosis. ${ }^{4,5}$ In terms of precipitating events, primary hepatic insult including reactivation of HBV as well as secondary viral etiologies such as HEV and HAV form a major cause in the east and APASL definition per se excludes bacterial infection as a precipitating event. ${ }^{6-8}$ On the other hand, infections as a group are significant precipitating events in data reported from the west. ${ }^{5}$ A recent EASL-CLIF consortium prospectively analyzed a large cohort of patients to specifically define various organ failures, to assess various factors predicting mortality and to develop a prognosticating model. ${ }^{5}$ The consortium also modified the existing SOFA score utilized by intensivists and devised a new CLIF-SOFA score to address various issues specifically associated with liver failure and cirrhosis. ${ }^{9}$ 
ACLF is frequently being recognized in clinical practice, so there is growing interest to understand the various aspects like the nature of acute insult, etiologies of underlying chronic liver diseases, pathophysiology, prognostic outcome and the role of supportive therapies. There is paucity of data from India; most studies are single-centre with limited sample size. Hence, this study was conducted to determine the primary etiologies and precipitating insults in patients with ACLF in Indian setting and to determine prognostic factors predicting the survival in these patients; with the help of CLIF - SOFA score.

\section{MATERIALS AND METHOD}

\section{Site \& Source of data:}

The present study was done in the Department of Medical Gastroenterology, PVS Memorial Hospital, Kochi, Kerala, India; over a period from January 2015 to June 2016

Sample Size and its criteria: The study enrolled 100 patients presenting at PVS Memorial Hospital, Kochi that fulfilled the below mentioned inclusion and exclusion criteria:

\section{Inclusion criteria:}

1. Patients diagnosed with uunderlying chronic liver disease

2. Serum bilirubin $>5 \mathrm{mg} / \mathrm{dl}$ and coagulopathy (INR more than 1.5 )

3. Ascites and/or encephalopathy

4. Patients with age 12 years or more.

\section{Exclusion criteria}

1. Patients with hepatic or extrahepatic malignancy.

2. Patients with portal vein thrombosis.

3. Patients with extraheatic biliary obstruction.

4. Patient who lost follow up after diagnosis

5. Patient that didn't gave consent for the study.

\section{Methodology:}

Diagnosis of ACLF was based on following criteria

1) Acute deterioration in liver function (over a period of $\leq 4$ weeks).

2) Manifesting as jaundice and coagulopathy with any of the following features

○ Ascites or

- Hepatic encephalopathy

3) Previously undiagnosed or diagnosed but compensated chronic liver disease.

Each patient was enrolled into the study after an informed consent taken. At the time of recruitment, each patient was personally interviewed to ascertain the information on demographic profile, history, standardized clinical examination, biochemical parameter and outcomes. Blood samples were collected for routine blood investigations, viral markers, 
autoimmune hepatitis marker (if required), Wilson disease panel (if required) and serum ferritin (if required). Upper GI endoscopy was performed using video endoscope to look for varices. Ultrasound abdomen examination was carried out in Department og Gastroenterology, by using 6-12 MHz linear array transducer with HDI 5000 sono, ALT, USG, Ultrasound system. CBC, blood sugar, Renal parameter, electrolytes, Liver function tests, ABG, serum ceruloplasmin (if required), serum ferritin (if required) and ascitic fluid of all the samples were estimated using an auto-analyzer. Urine examination was obtained by urin analyzer and light microscopy. HBsAg, IgM anti-HBc, HBeAg, Anti-HCV, IgM anti-HEV and IgM anti HAV were detected with enzyme immunoassay. Hepatitis B virus-DNA was detected by means of polymerase chain reaction (PCR) using primers specific for the $\mathrm{S}$ and pre-C/C regions of the HBV genome. Serum HBV-DNA levels were quantified using a branched- DNA assay. In addition to HCV RNA and genotype were assessed by standard method.

\section{Management protocol:}

All patients were managed with standard of care therapy as per decision of treating physician. Patients with hepatitis B are started on antiviral drugs (tenofovir or entecavir). Renal replacement therapy was provided as required. Management of upper GI bleed, hepatorenal syndrome, spontaneous bacterial peritonitis, hepatic encephalopathy and other infections were as per the guidelines followed in the unit. Patients with variceal bleeding underwent endoscopic variceal ligation. Spontaneous bacterial peritonitis and hepatorenal syndrome were managed as per recommendations. Rifaximin and lactulose are started in patients with hepatic encephalopathy. Need for ventilator support as well as the need for and choice of antibiotics were decided by the treating clinicians. Patients are followed up during hospital stay and outcome was noted at 28 day. In case of worsening ACLF patient were considered for liver transplant. However, none of the patients underwent liver transplantation.

\section{Data collection and Statistical Methods:}

The data of following variables was collected at admission: age, gender, clinical presentation, laboratory parameters (hemogram, liver function test, RFT, electrolytes, International Normalized Ratio), CTP score, MELD, MELD Na, SOFA, APACHE II score, CLIF-SOFA score at baseline. Different cultures were obtained. In addition, cause of acute hepatic decompensation, etiology of underlying CLD and outcomes were noted. Mean duration of their hospital stay was 2 weeks. We assessed the in-hospital mortality and outcomes at 28 days.

Descriptive and inferential statistical analysis has been carried out in the present study. Results on continuous measurements were presented on Mean \pm SD (Min-Max) and results on categorical measurements were presented in Number (\%). Significance is assessed at $5 \%$ level of significance. Mean values were compared between survivors and non survivors. Univariate and multivariate analysis of all relevant clinical and biochemical variable was performed at 
admission. The odds ratio (OR) with $95 \%$ confidence interval and the diagnostic accuracy of each predictor was also calculated. The following assumptions on data was made,

\section{Assumptions:}

1. Dependent variables should be normally distributed.

2. Samples drawn from the population should be random.

3. Cases of the samples should be independent.

Analysis of variance (ANOVA) was used to find the significance of study parameters between three or more groups of patients. Student $t$ test (two tailed, independent) has been used to find the significance of study parameters on continuous scale between two groups (Inter group analysis) on parametric parameters. Chi-square/ Fisher Exact test has been used to find the significance of study parameters on categorical scale between two or more groups for nonparametric setting for Qualitative data analysis. The Statistical software namely SAS 9.2, SPSS 15.0, Stata 10.1, MedCalc 9.0.1, Systat 12.0 and R environment ver.2.11.1 were used for the analysis of the data and MS Word and MS Excel have been used to generate graphs and tables.

\section{Significant figures:}

** Strongly significant ( $\mathrm{p}$ value: $\mathrm{p} \leq 0.01$ )

* Moderately significant ( $\mathrm{p}$ value: $0.01<\mathrm{p} \leq 0.05$ )

+ Suggestive significance ( $\mathrm{p}$ value: $0.05<\mathrm{p}<0.10$ )

NS- Non-significant ( $p$ value: $p>0.1$ )

\section{RESULTS AND DISCUSSION}

Out of 100 patients studied, 87 were males and 17 females with sex ratio 4.9:1. The mean age of all subjects was $54.21 \pm 11.23$ years. Alcohol was the primary etiology for cirrhosis in $69 \%$ patients while $25 \%$ patients etiology could be identified and labelled as NASH/Cryptogenic. Among identifiable causes viral related CLD etiology, HBV infection was seen in $2 \%$ patients and HCV infection was observed in only one patient. Other cause of CLD was autoimmune hepatitis recorded in $3 \%$ patients. Superadded HAV and HEV infection was seen in $2 \%$ and $5 \%$ patients respectively that accounted to acute decompensation. In our study, autoimmune hepatitis related CLD was seen in 3\% patients and acute decompensation due to flare of their autoimmune liver disease was seen in $2 \%$ cases. Bacterial infections and upper GI bleed were the precipitating events in $9 \%$ and $10 \%$ of the patients respectively. Around $30 \%$ patients had $>1$ precipitating event while in $6 \%$ precipitating event was drugs related. Trauma and surgery related precipitating events was seen $2 \%$ patients. Jaundice (100\%) and abdominal distension $(100 \%)$ were the most frequent symptoms reported by the patients with ACLF in our study. Other symptoms were anorexia (62\%), fatigability (45\%), fever (50\%) and pain abdomen (16\%). 51\% patients had history of significant alcohol intake while $71 \%$ patient had history of 
pedal edema. Moreover, 10\% patients had history of GI bleed. 36\% patients were in grade I and II hepatic encephalopathy at the time of admission respectively. 13\% had grade 3-4 hepatic encephalopathy. Liver, renal, coagulation, cerebral, circulatory and respiratory failure were seen in $67 \%, 32 \%, 39 \%, 15 \%, 39 \%, 30 \%$, and $13 \%$ respectively. Most frequent complication was coagulopathy i.e. INR $>2.5$, which was found in $36 \%$ of patients, followed by raised creatinine ( $>2 \mathrm{mg} / \mathrm{dl})$ in $31 \%$ of patients. Hemoglobin, platelet, INR, and serum creatinine were factors which had a significant association with ACLF group as per CLIF-SOFA. There were no significant differences in total count, serum sodium, serum potassium, total bilirubin, albumin, AST, ALT and ALP with ACLF group. The most striking differences were seen in MELD, MELD Na, APACHE II and SOFA scores between no ACLF and ACLF group. Among all four scores, APACHE II had highest sensitivity of $92.7 \%$ ( $\mathrm{p}<0.001)$. Mortality at 28 days was recorded in 55\% patients. Grade I-II varices were seen in $42 \%$ patients and grade III-IV varices in $58 \%$ patients. Sepsis was seen in $34 \%$ patients and SBP in $23 \%$ patients. UTI, cellulitis and pneumonia were observed in 13\%, $7 \%$ and $20 \%$ respectively. Mortality was highest in ACLF group II (77.8\%) and ACLF group III was (95.7\%).

In this prospective observational study, data of ACLF patients diagnosed as per the APASL criteria and Organ failures (OF) as per the Chronic Liver Failure- Sequential Organ Failure Assessment (CLIF-SOFA) was assessed. Prognostic scores including Child Turcotte Pugh (CTP) score, model for end stage liver disease (MELD), model for end stage liver disease (MELD) - sodium (Na), sequential organ failure assessments (SOFA) score and acute physiology and chronic health evaluation score (APACHE II) were calculated as per previously defined criteria. In addition, patients with extra-hepatic acute insults such as sepsis and variceal bleed were also included. The primary objective of the study was to evaluate the clinical profile, precipitating factors, course and outcome of ACLF patients at 28 days. This study is comparable to various Indian and International studies with minor differences, probably due to distinct regional differences in etiological profile and variation in sample size. The study was concluded with 100 patients within the speculated time period.

In the present study, median age of presentation ranging was from 43 to 65 years that is similar to Acharya et al. ${ }^{10}$ and Radha Krishna et al. ${ }^{7}$ Males predominated in our study by 2.7 times and was similar to male predominance from 2.3 to 3 times that had been observed by Duseja et al. ${ }^{11}$ However, a study from European country reported older median age of presentation (57 years), this might be attributed to late onset of cirrhosis in their population. ${ }^{12}$ Around $69 \%$ of our patients had alcohol as primary etiology of cirrhosis while $25 \%$ belonged to cryptogenic/ NASH category, similar to a study done by Amarapurkar et al. ${ }^{13}$ and Duseja et al. ${ }^{11}$ Alcohol was reported as the most common etiology of cirrhosis in the CANONIC study(49.2\%). ${ }^{12}$ In present study, alcohol was found to be the most common etiology of CLD (69\%). This contrasts 
with previous studies from India where the prevalence of alcoholic cirrhosis was $10 \%$ to 30 $\%$. Studies by Garg et al. and Krishna et al. had $30 \%$ to $64 \%$ of patients with ACLF belonging to HBV-associated cirrhosis. . $^{6,7}$

Reactivation of HBV has been a predominant cause of acute-on-chronic liver failure presentation in most studies from Asia-Pacific region. However, we had 2\% (2 patients) of HBV reactivation on a background of HBV cirrhosis and was comparable to a study done by Duseja et al. ${ }^{11}$ Similarly, acute viral hepatitis insults like HBV (15\%), HEV (5\%) and HAV (5\%) were markedly seen from our study, though they have been well reported in literature..$^{6,7,10,11}$

In present study, HBV infection was the most common in contrast to HEV infection, this explains hyper-endemicity of HBV in South India. Active alcoholism was present in almost an half of our patients presenting as ACLF, which correlates well with another study by Garg et al. where alcohol hepatitis was present in $27 \%$ of their patients. ${ }^{6}$ In present study, there was a significantly high 28-day mortality rate of $55 \%$ in patients with ACLF by APASL criteria, which was comparable to similar studies by Duseja et al. (mortality of $46 \%$ ) and Amarapurkar et al. (mortality of $43.1 \%$ ) which had a heterogeneous study population. ${ }^{11,13}$

\section{ACLF by EASL-CLIF consortium criteria}

We then reclassified our patients into ACLF grades as defined by the EASL- CLIF consortium under the CANONIC study. In present study, presence of single organ failure carried a similar mortality rate as those with no organ failure (10\%), but mortality rate exceeded $77.8 \%$ in those with two organ failures, whereas it was $99.5 \%$ in those with three or more organ failures. Our study showed higher mortality (55\%) as compared to Amarapurkar et al. ${ }^{13}(33.87 \%)$ and Moreau et al. ${ }^{12}(>15 \%)$ which can be attributed to heterogenous population.

\begin{tabular}{|c|c|c|c|c|}
\hline $\begin{array}{l}\text { No. Organ } \\
\text { Failure }\end{array}$ & $\begin{array}{l}\text { Prevalence } \\
\text { (In present } \\
\text { study)(n=100) }\end{array}$ & $\begin{array}{l}\text { Mortality (In } \\
\text { present study) } \\
(\mathbf{n}=100)\end{array}$ & $\begin{array}{l}\text { Prevalence } \\
\text { (Amarapurkar } \\
\left.\text { et } \text { al. }^{13}\right) \quad(n=62)\end{array}$ & $\begin{array}{l}\text { Mortality } \\
\text { (Amarapurkar } \\
\text { et al.13)( } n=62 \text { ) }\end{array}$ \\
\hline No organ failure & $30(30 \%)$ & $3 / 30(10 \%)$ & $12(19.3 \%)$ & $1 / 12(8.3 \%)$ \\
\hline Organ failure & $70(70 \%)$ & $52 / 70(74.28 \%)$ & $50(80.6 \%)$ & $20 / 50(40 \%)$ \\
\hline 1 organ failure & $20(20 \%)$ & $9 / 20(45 \%)$ & $24(38.7 \%)$ & $2 / 24(8.3 \%)$ \\
\hline 2 organ failure & $27(27 \%)$ & $21 / 27(77.8 \%)$ & $11(17.7 \%)$ & $6 / 11(54.5 \%)$ \\
\hline 3 organ failure & $23(23 \%)$ & $22 / 23(95.7 \%)$ & $15(24.1 \%)$ & $12 / 15(80 \%)$ \\
\hline
\end{tabular}

Similarly, presence of individual organ failure (liver, renal, coagulation, cerebral, circulatory, and lung) was determined and correlated with mortality. In present study, OF's liver, renal, coagulation, cerebral, circulatory and respiratory failure were seen in $67 \%, 32 \%, 39 \%, 15 \%$, $39 \%, 30 \%$, and $13 \%$ respectively. On the other hand, Amarapurkar et al. ${ }^{13}$ in its study reported OF's liver, renal, coagulation, cerebral, circulatory and respiratory failure in $20.9 \%, 25.8 \%$, $14.5 \%, 62.9 \%, 29 \%$ respectively. In present study, higher percentage of organ failures and 
related mortality was seen as compared to Amarapurkar et al. ${ }^{13}$ because of heterogeneous population and large sample size.

\begin{tabular}{lllll}
\hline $\begin{array}{l}\text { Organ } \\
\text { Failure }\end{array}$ & $\begin{array}{l}\text { Prevalence (In } \\
\text { present } \\
\text { study)(n=100) }\end{array}$ & $\begin{array}{l}\text { Mortality (In } \\
\text { present study) } \\
(\mathbf{n = 5 5})\end{array}$ & $\begin{array}{l}\text { Prevalence (In } \\
\text { Amarapurkar et } \\
\left.\text { al. }^{\mathbf{1 3}}\right)(\mathbf{n = 6 2})\end{array}$ & $\begin{array}{l}\text { Mortality( In } \\
\text { Amarapurkar et } \\
\left.\text { al. }^{\mathbf{1 3}}\right)(\mathbf{n = 2 1})\end{array}$ \\
\hline Liver & $67(67 \%)$ & $37 / 67(55.22 \%)$ & $13(20.9 \%)$ & $5 / 13(38.45 \%)$ \\
Kidney & $32(32 \%)$ & $28 / 32(87.5 \%)$ & $16(25.8 \%)$ & $10 / 16(62.5 \%)$ \\
Cerebral & $15(15 \%)$ & $13 / 15(86.66 \%)$ & $39(62.9 \%)$ & $19 / 39(48.7 \%)$ \\
Coagulation & $39(39 \%)$ & $31 / 39(79.48 \%)$ & $9(14.5 \%)$ & $7 / 9(77.7 \%)$ \\
Circulatory & $30(30 \%)$ & $28 / 30(93.3 \%)$ & $18(29 \%)$ & $13 / 18(72.2 \%)$ \\
Respiratory & $13(13 \%)$ & $12 / 13(92.3 \%)$ & & \\
\hline
\end{tabular}

\section{Comparison of patients across ACLF grades}

In present study, when patients were compared across ACLF grades with those without ACLF on same parameters as previously, same factors were found to be significant; e.g. presence of organ failure other than liver failure; Hemoglobin, platelet, INR, serum creatinine, MELD, MELD Na, APACHE II, SOFA and CLIF-SOFA. Those with ACLF grades 2 and 3 had infection as a significantly higher proportion as a precipitating event. Prevalence of all individual organ failures except liver failure was significantly higher in ACLF grade 3 patients as compared to other ACLF grades or no ACLF patients. Thus, there was clearly an increasing trend across no ACLF, ACLF 1, ACLF 2 and ACLF 3 in INR, serum creatinine, MELD, MELD-Na, APACHE II and SOFA scores. These results are comparable to Amarapurkar et al. ${ }^{13}$ (Except for the group ACLF 1 where total number of patients were small) and Moreau et al. ${ }^{12}$

As per the EASL-CLIF criteria, we divided the patients into no ACLF and ACLF. ACLF further graded as 1,2, and 3 according to presence of organ failure and severity of renal and cerebral failure. Prevalence of infection as a precipitating event was significantly higher in ACLF group, as also presence of $>1$ precipitating event. This corresponds very well to similar study by Moreau et al. ${ }^{12}$ where these parameters were statistically very significant, though they also found active alcoholism as a significant factor which was also seen in our analysis. In terms of organ failure, definitely the ACLF group had significant prevalence of organ failure excluding liver failure across all grades of ACLF. Hemoglobin, platelet, INR, and serum creatinine were factors which had a significant association with ACLF group. This correlates well with study by Moreau et al. ${ }^{12}$ and Amarapurkar et al. ${ }^{13}$ except for raised total leukocyte count. While raised serum creatinine suggests important contribution of renal failure in the high mortality associated with the ACLF syndrome. Mortality rates were $80 \%$ in ACLF patients, signifying significantly high mortality as number of organ failures increase. The most higher significance prognostic scores MELD, MELD-Na, SOFA and AACHE II were seen in ACLF groups $(\mathrm{p}<0.001)$.CLIF-SOFA and APACHE II score had a higher sensitivity in predicting 
mortality as compared to MELD, MELD-Na, SOFA and CTP score, though some of the previous studies have found MELD score to be equally good in predicting mortality. ${ }^{14}$

\section{Comparison of survivors and non-survivors}

Non-survivors had significantly raised INR and serum creatinine as compared survivors $(\mathrm{p}<0.001)$. Hemoglobin, platelet and Albumin levels were significantly lower in non-survivors as compared to survivors $(\mathrm{p}<0.001)$. Alcohol related chronic liver disease and continuous alcohol consumption was seen in a significantly higher proportion of non-survivors and infection was more frequent $(\mathrm{p}<0.001)$. The various prognostic scores- CTP, SOFA, APACHEII, MELD and MELD Na were significantly higher in non-survivors. There were no significant differences in age, sex, total count, serum sodium, serum potassium, tostal bilirubin, AST, ALT and ALP. Organ failures (Renal, cerebral, coagulation, circulatory and respiratory) were significantly higher in non survivors as compared to survival $(\mathrm{p}<0.001)$. The most striking differences were seen in serum creatinine, INR, CTP, MELD, MELD Na, SOFA, APACHE II and CLIF-SOFA scores between survivors and non-survivors. Also, hepatic causes of acute insult were higher in survivors, probably implying that systemic inflammation in these patients may have been lesser.

Table 1: Baseline characteristics \& their respective distribution.

\begin{tabular}{lcc}
\hline $\begin{array}{l}\text { Variables } \\
\text { Gender }\end{array}$ & Patient (n) & Percentage (\%) \\
\hline Male & 17 & 17.0 \\
Female & 83 & 83.0 \\
Age (years) & & \\
$<30$ & 1 & 1.0 \\
$31-40$ & 11 & 11.0 \\
$41-50$ & 28 & 28.0 \\
$51-60$ & 33 & 33.0 \\
$61-70$ & 19 & 19.0 \\
$71-80$ & 6 & 6.0 \\
$>80$ & 2 & 2.0 \\
Underlying cause of chronic liver disease & \\
Alcohol & 69 & 69.0 \\
HBV & 2 & 2.0 \\
NASH/Cryptogenic & 25 & 25.0 \\
Autoimmune disease & 3 & 3.0 \\
HCV & 1 & 1.0 \\
Precipitating events in Various Subgroups of CLD \\
Active alcoholism & 51 & 51.0 \\
Acute viral hepatitis (HAV) & 2 & 2 \\
Acute viral hepatitis (HEV) & 5 & 5 \\
Acute viral hepatitis (HBV) & 15 & 15 \\
Reactivation HBV & 2 & 2 \\
HCV & 2 & 2 \\
Upper GI Bleed & 10 & 10.0 \\
Bacterial Infection & 9 & 9.0 \\
Drugs & 6 & 6.0 \\
\hline
\end{tabular}




\begin{tabular}{|c|c|c|}
\hline Flare of autoimmune & 2 & 2.0 \\
\hline Trauma/Surgery & 2 & 2.0 \\
\hline Others & 6 & 6.0 \\
\hline$>1$ Precipitating factors & 30 & 30 \\
\hline \multicolumn{3}{|l|}{ Clinical Profile } \\
\hline Anorexia & 66 & 66.0 \\
\hline Fatigue & 45 & 45.0 \\
\hline Jaundice & 100 & 100. \\
\hline Fever & 50 & 50.0 \\
\hline Abdomen pain & 16 & 16.0 \\
\hline GI bleed & 10 & 10.0 \\
\hline Active alcohol & 51 & 53.0 \\
\hline Abdomen distension (Ascites) & 100 & 100. \\
\hline Pedal edema & 73 & 73.0 \\
\hline HE ( Grade I/II ) & 36 & 36.0 \\
\hline HE ( Grade III/IV ) & 13 & 13. \\
\hline Drugs intake & 6 & 6.0 \\
\hline Others & 5 & 5.0 \\
\hline \multicolumn{3}{|l|}{ Organ Failure } \\
\hline Liver & 67 & 67.0 \\
\hline Kidney & 32 & 32.0 \\
\hline Cerebral & 15 & 15.0 \\
\hline Coagulation & 39 & 39.0 \\
\hline Circulatory & 30 & 30.0 \\
\hline Respiratory & 13 & 13.0 \\
\hline \multicolumn{3}{|l|}{ INR level } \\
\hline $1.5-2$ & 31 & 31. \\
\hline $2.1-2.5$ & 33 & $33 .($ \\
\hline$>2.5$ & 36 & $36 .($ \\
\hline \multicolumn{3}{|l|}{ Serum creatinine (mg/dl) } \\
\hline$<1.5$ & 48 & 48. \\
\hline $1.5-2$ & 21 & 21. \\
\hline$>2$ & 31 & $31 .($ \\
\hline \multicolumn{3}{|l|}{ Sodium (mEq/l) } \\
\hline$<130$ & 93 & 93. \\
\hline$>130$ & 6 & 6.0 \\
\hline
\end{tabular}

ACLF Grade distribution as per EASL-CLIF consortium

Group 0

Group 1

30

30.0

Group 2

$20 \quad 20.0$

Group 3

27.0

Final Outcome

$\begin{array}{lll}\text { Sepsis } & 34 & 34.0\end{array}$

$\begin{array}{lll}\text { SBP } & 23 & 23.0\end{array}$

Cellulitis $\quad 7 \quad 7.0$

UTI $13 \quad 13.0$

Pneumonia $20 \quad 20.0$

Mortality $\quad 55 \quad 55.0$

Table 2: Comparison of Clinical features of ACLF patients in relation to mortality.

\begin{tabular}{lllll}
\hline $\begin{array}{l}\text { Clinical } \\
\text { Profile }\end{array}$ & $\begin{array}{l}\text { Non } \\
\text { survival(n=55) }\end{array}$ & $\begin{array}{l}\text { Survival } \\
(\mathbf{n = 4 5})\end{array}$ & $\begin{array}{l}\text { Total } \\
(\mathbf{n = 1 0 0})\end{array}$ & P value \\
\hline Anorexia & $36(65.5 \%)$ & $30(66.7 \%)$ & $66(66 \%)$ & 0.899 \\
Fatigue & $29(52.7 \%)$ & $16(35.6 \%)$ & $45(45 \%)$ & $0.086+$ \\
\hline
\end{tabular}




\begin{tabular}{lllll}
\hline Jaundice & $55(100 \%)$ & $45(100 \%)$ & $100(100 \%)$ & 1.000 \\
Fever & $36(65.5 \%)$ & $14(31.1 \%)$ & $50(50 \%)$ & $0.001^{* *}$ \\
Abdomen pain & $11(20 \%)$ & $5(11.1 \%)$ & $16(16 \%)$ & 0.228 \\
GI bleed & $23(41.8 \%)$ & $10(22.2 \%)$ & $33(33 \%)$ & $0.038^{*}$ \\
Active alcohol & $27(49.1 \%)$ & $26(57.8 \%)$ & $53(53 \%)$ & 0.387 \\
Ascites & $55(100 \%)$ & $45(100 \%)$ & $100(100 \%)$ & 1.000 \\
Pedal edema & $41(74.5 \%)$ & $32(71.1 \%)$ & $73(73 \%)$ & 0.700 \\
\hline
\end{tabular}

Table 3: Comparison of lab parameters of ACLF patients in relation to mortality.

\begin{tabular}{lllll}
\hline Lab. variables & $\begin{array}{l}\text { Non survival } \\
(\mathbf{n = 5 5})\end{array}$ & $\begin{array}{l}\text { Survival } \\
(\mathbf{n = 4 5})\end{array}$ & Total & P value \\
\hline Hemoglobin $(\mathrm{g} / \mathrm{dl})$ & $9.08 \pm 2.39$ & $10.69 \pm 1.97$ & $9.81 \pm 2.34$ & $<0.001^{* *}$ \\
Total Count (dl) & $13818.18 \pm 4752.04$ & $12957.78 \pm 11219.07$ & $13431.00 \pm 8273.06$ & 0.607 \\
Platelet Count & $93543.64 \pm 60245.88$ & $123168.89 \pm 73983.71$ & $106875.00 \pm 68057.94$ & $0.030^{*}$ \\
Bilirubin (mg/dl) & $16.46 \pm 8.21$ & $15.93 \pm 8.14$ & $16.22 \pm 8.14$ & 0.745 \\
ALT-U/L & $264.46 \pm 638.72$ & $256.14 \pm 405.80$ & $260.71 \pm 543.81$ & 0.940 \\
AST-U/L & $493.59 \pm 1351.1$ & $328.73 \pm 401.69$ & $419.40 \pm 036.44$ & 0.432 \\
ALP-U/L & $95.35 \pm 33.15$ & $105.98 \pm 47.74$ & $100.14 \pm 40.50$ & 0.193 \\
Albumin-gm/dl & $2.53 \pm 0.46$ & $2.83 \pm 0.45$ & $2.67 \pm 0.48$ & $0.002^{* *}$ \\
INR & $2.70 \pm 0.72$ & $2.18 \pm 0.57$ & $2.46 \pm 0.70$ & $<0.001^{* *}$ \\
Serum & $2.05 \pm 1.24$ & $1.09 \pm 0.71$ & $1.62 \pm 1.14$ & $<0.001^{* *}$ \\
Creatinine(mg/dl) & & & & \\
Sodium (mEq/l) & $124.51 \pm 6.89$ & $126.96 \pm 6.67$ & $125.61 \pm 6.87$ & $0.076+$ \\
\hline
\end{tabular}

Table 4: Organ Failure distribution of ACLF patients in relation to mortality.

\begin{tabular}{lllll}
\hline Organ Failure & $\begin{array}{l}\text { Non survival } \\
(\mathbf{n = 5 5})\end{array}$ & $\begin{array}{l}\text { Survival } \\
(\mathbf{n = 4 5})\end{array}$ & $\begin{array}{l}\text { Total } \\
(\mathbf{n = 1 0 0})\end{array}$ & P value \\
\hline Liver & $37(67.3 \%)$ & $30(66.7 \%)$ & $67(67 \%)$ & 0.949 \\
Kidney & $28(50.9 \%)$ & $4(8.9 \%)$ & $32(32 \%)$ & $<0.001^{* *}$ \\
Cerebral & $13(23.6 \%)$ & $2(4.4 \%)$ & $15(15 \%)$ & $0.007^{* *}$ \\
Coagulation & $31(56.4 \%)$ & $8(17.8 \%)$ & $39(39 \%)$ & $<0.001^{* *}$ \\
Circulatory & $28(50.9 \%)$ & $2(4.4 \%)$ & $30(30 \%)$ & $<0.001^{* *}$ \\
Respiratory & $12(21.8 \%)$ & $1(2.2 \%)$ & $13(13 \%)$ & $0.004^{* *}$ \\
\hline
\end{tabular}

Table 5: Comparison of MELD, MELD-Na, SOFA and APACHE-II of ACLF patients in relation to mortality.

\begin{tabular}{lllll}
\hline Variables & Mortality & \multicolumn{2}{l}{ Total } & P value \\
\cline { 2 - 4 } & Yes & No & & \\
\hline MELD & $32.84 \pm 4.74$ & $26.40 \pm 3.80$ & $29.94 \pm 5.39$ & $<0.001^{* *}$ \\
MELD Na & $34.96 \pm 3.28$ & $30.31 \pm 2.91$ & $32.87 \pm 3.88$ & $<0.001^{* *}$ \\
SOFA & $10.78 \pm 3.22$ & $6.20 \pm 2.39$ & $8.72 \pm 3.67$ & $<0.001^{* *}$ \\
APACHE II & $25.07 \pm 6.03$ & $14.51 \pm 5.72$ & $20.32 \pm 7.89$ & $<0.001^{* *}$ \\
\hline
\end{tabular}

Table 6: Sepsis, SBP, cellulites, Pneumonia and UTI in ACLF patients in relation to incidence of mortality.

\begin{tabular}{lllll}
\hline Outcomes & $\begin{array}{l}\text { Non survival } \\
(\mathbf{n = 5 5})\end{array}$ & $\begin{array}{l}\text { Survival } \\
(\mathbf{n = 4 5})\end{array}$ & $\begin{array}{l}\text { Total } \\
(\mathbf{n = 1 0 0})\end{array}$ & P value \\
\hline Sepsis & $30(54.5 \%)$ & $4(8.9 \%)$ & $34(34 \%)$ & $<0.001^{* *}$ \\
SBP & $13(23.6 \%)$ & $10(22.2 \%)$ & $23(23 \%)$ & 0.867 \\
Cellulites & $4(7.3 \%)$ & $3(6.7 \%)$ & $7(7 \%)$ & 1.000 \\
Pneumonia & $18(32.7 \%)$ & $2(4.4 \%)$ & $20(20 \%)$ & $<0.001^{* *}$ \\
UTI & $9(16.4 \%)$ & $4(8.9 \%)$ & $13(13 \%)$ & 0.269 \\
\hline
\end{tabular}


Table 7: Distribution of patients studied in relation to incidence of mortality.

\begin{tabular}{lllll}
\hline Variables & Non survival(n=55) & $\begin{array}{l}\text { Survival } \\
(\mathbf{n = 4 5})\end{array}$ & Total & Significance \\
\hline $\begin{array}{l}\text { Gender } \\
\text { Female }\end{array}$ & $9(52.94 \%)$ & $8(47.05 \%)$ & $17(17 \%)$ & $\mathrm{p}=1.00(\mathrm{NS})$ \\
$\begin{array}{l}\text { Male } \\
\text { Age (years) }\end{array}$ & & & & \\
$<30$ & $1(1.8 \%)$ & $37(38.55 \%)$ & $83(83 \%)$ & \\
$31-40$ & $4(7.3 \%)$ & $0(0 \%)$ & $1(1 \%)$ & $\mathrm{p}=0.90(\mathrm{NS})$ \\
$41-50$ & $19(34.5 \%)$ & $7(15.6 \%)$ & $11(11 \%)$ & \\
$51-60$ & $17(30.9 \%)$ & $9(20 \%)$ & $28(28 \%)$ & \\
$61-70$ & $9(16.4 \%)$ & $16(35.6 \%)$ & $33(33 \%)$ & \\
$71-80$ & $4(7.3 \%)$ & $10(22.2 \%)$ & $19(19 \%)$ & \\
$>80$ & $1(1.8 \%)$ & $2(4.4 \%)$ & $6(6 \%)$ & \\
\hline
\end{tabular}


Table 8: Distribution of patients studied in relation to ACLF groups as per CLIF-SOFA score.

\begin{tabular}{|c|c|c|c|c|c|}
\hline \multirow[t]{2}{*}{ Variable } & \multicolumn{4}{|l|}{ ACLF Group } & \multirow[t]{2}{*}{ Total } \\
\hline & Group 0 & Group I & Group II & Group III & \\
\hline \multicolumn{6}{|l|}{ Gender } \\
\hline Female & $7(23.3 \%)$ & $4(20 \%)$ & $2(7.4 \%)$ & $4(17.4 \%)$ & \multirow[t]{2}{*}{$0.413(\mathrm{NS})$} \\
\hline Male & $23(76.7 \%)$ & $16(80 \%)$ & $25(92.6 \%)$ & $19(82.6 \%)$ & \\
\hline \multicolumn{6}{|l|}{ Age (years) } \\
\hline$<30$ & $0(0 \%)$ & $0(0 \%)$ & $0(0 \%)$ & $1(4.3 \%)$ & \multirow[t]{7}{*}{$0.915(\mathrm{NS})$} \\
\hline $31-40$ & $5(16.7 \%)$ & $2(10 \%)$ & $3(11.1 \%)$ & $1(4.3 \%)$ & \\
\hline $41-50$ & $8(26.7 \%)$ & $5(25 \%)$ & $6(22.2 \%)$ & $9(39.1 \%)$ & \\
\hline $51-60$ & $11(36.7 \%)$ & $6(30 \%)$ & $9(33.3 \%)$ & $7(30.4 \%)$ & \\
\hline $61-70$ & $4(13.3 \%)$ & $5(25 \%)$ & $6(22.2 \%)$ & $4(17.4 \%)$ & \\
\hline $71-80$ & $1(3.3 \%)$ & $2(10 \%)$ & $2(7.4 \%)$ & $1(4.3 \%)$ & \\
\hline$>80$ & $1(3.3 \%)$ & $0(0 \%)$ & $1(3.7 \%)$ & $0(0 \%)$ & \\
\hline \multicolumn{6}{|l|}{ Laboratory Parameters } \\
\hline Hemoglobin $(\mathrm{g} / \mathrm{dl})$ & $11.13 \pm 1.90$ & $9.94 \pm 2.14$ & $9.09 \pm 2.33$ & $8.81 \pm 2.35$ & $0.001 * *$ \\
\hline Total Count (dl) & $13486.67 \pm 13155.32$ & $12215.00 \pm 5302.06$ & $13125.93 \pm 4014.84$ & $14773.91 \pm 5761.25$ & $0.788(\mathrm{NS})$ \\
\hline Platelet Count & $146166.67 \pm 77083.96$ & $102520.00 \pm 79805.27$ & $93548.15 \pm 44327.4$ & $75056.52 \pm 42302.17$ & $0.001 * *$ \\
\hline Bilirubin $(\mathrm{mg} / \mathrm{dl})$ & $17.27 \pm 8.78$ & $14.42 \pm 8.46$ & $15.79 \pm 7.83$ & $16.92 \pm 7.55$ & $0.638(\mathrm{NS})$ \\
\hline ALT-U/L & $178.71 \pm 297.03$ & $318.85 \pm 405.43$ & $204.07 \pm 374.44$ & $383.61 \pm 935.34$ & $0.504(\mathrm{NS})$ \\
\hline AST-U/L & $248.87 \pm 319.41$ & $477.55 \pm 491.89$ & $277.26 \pm 394.89$ & $758.14 \pm 2029.47$ & $0.282(\mathrm{NS})$ \\
\hline ALP-U/L & $107.28 \pm 48.66$ & $103.10 \pm 44.77$ & $101.41 \pm 35.81$ & $86.76 \pm 27.36$ & $0.314(\mathrm{NS})$ \\
\hline Albumin-gm/dl & $2.87 \pm 0.44$ & $2.65 \pm 0.48$ & $2.58 \pm 0.48$ & $2.52 \pm 0.45$ & $0.029 *$ \\
\hline INR & $2.09 \pm 0.48$ & $2.33 \pm 0.57$ & $2.57 \pm 0.76$ & $2.95 \pm 0.69$ & $<0.001 * *$ \\
\hline Serum Creatinine $(\mathrm{mg} / \mathrm{dl})$ & $0.81 \pm 0.31$ & $1.71 \pm 0.97$ & $1.79 \pm 0.89$ & $2.39 \pm 1.54$ & $<0.001 * *$ \\
\hline Sodium $(\mathrm{mEq} / \mathrm{l})$ & $126.10 \pm 5.00$ & $126.20 \pm 5.80$ & $126.04 \pm 9.13$ & $123.96 \pm 6.93$ & $0.635(\mathrm{NS})$ \\
\hline \multicolumn{6}{|l|}{ CTP score } \\
\hline $\mathrm{B}$ & $5(16.7 \%)$ & $1(5 \%)$ & $0(0 \%)$ & $0(0 \%)$ & \multirow[t]{2}{*}{$0.019^{*}$} \\
\hline $\mathrm{C}$ & $25(83.3 \%)$ & $19(95 \%)$ & $27(100 \%)$ & $23(100 \%)$ & \\
\hline \multicolumn{6}{|l|}{ Prognostic score } \\
\hline MELD & $25.07 \pm 3.26$ & $29.95 \pm 3.22$ & $31.48 \pm 4.96$ & $34.48 \pm 4.65$ & $<0.001 * *$ \\
\hline
\end{tabular}




\begin{tabular}{|l|l|l|l|l|l|}
\hline MELD Na & $29.30 \pm 2.83$ & $32.95 \pm 2.16$ & $33.74 \pm 3.32$ & $36.43 \pm 2.90$ & $<0.001^{* *}$ \\
\hline SOFA & $5.53 \pm 1.78$ & $7.70 \pm 2.30$ & $9.85 \pm 2.44$ & $12.43 \pm 3.74$ & $<0.001^{* *}$ \\
\hline APACHE II & $12.33 \pm 3.70$ & $19.65 \pm 5.68$ & $24.04 \pm 6.00$ & $26.96 \pm 6.41$ & $<0.001^{* *}$ \\
\hline Infections & $0(0 \%)$ & $2(10 \%)$ & $12(44.4 \%)$ & $6(26.1 \%)$ & $<0.001 * *$ \\
\hline Pneumonia & $1(3.3 \%)$ & $3(15 \%)$ & $3(11.1 \%)$ & $6(26.1 \%)$ & $0.098+$ \\
\hline UTI & $3(10 \%)$ & $1(5 \%)$ & $2(7.4 \%)$ & $1(4.3 \%)$ & $0.504(\mathrm{NS})$ \\
\hline Cellulites & $5(16.7 \%)$ & $5(25 \%)$ & $10(37 \%)$ & $14(60.9 \%)$ & $0.07+$ \\
\hline Sepsis & $7(23.3 \%)$ & $7(35 \%)$ & $5(18.5 \%)$ & $4(17.4 \%)$ & $0.910(\mathrm{NS})$ \\
\hline SBP
\end{tabular}


Table 9: The mortality at 28 days studied in relation to ACLF group as per CLIF-

SOFA.

\begin{tabular}{llllll}
\hline Outcomes & ACLF Group & & \multicolumn{2}{c}{ Total } \\
& Group 0 & Group I & Group II & Group III & \\
\hline Non survival & $3(10 \%)$ & $9(45 \%)$ & $21(77.8 \%)$ & $22(95.7 \%)$ & $55(55 \%)$ \\
Survival & $27(90 \%)$ & $11(55 \%)$ & $6(22.2 \%)$ & $1(4.3 \%)$ & $45(45 \%)$ \\
Total & $30(100 \%)$ & $20(100 \%)$ & $27(100 \%)$ & $23(100 \%)$ & $100(100 \%)$ \\
\hline
\end{tabular}

Table 10: ROC curve analysis to predict mortality.

\begin{tabular}{lllllllll}
\hline \multirow{2}{*}{ Variables } & \multicolumn{3}{l}{ ROC results to predict Mortality } & \multicolumn{2}{c}{ Cut-off } & AUROC & SE & P value \\
\cline { 2 - 5 } & Sensitivity & Specificity & LR+ & LR- & & & & \\
\hline CTP & 69.09 & 80.00 & 3.45 & 0.39 & $>12$ & 0.787 & 0.045 & $<0.001^{* *}$ \\
MELD & 85.45 & 73.33 & 3.20 & 0.20 & $>28$ & 0.856 & 0.037 & $<0.001^{* *}$ \\
MELD-Na & 74.55 & 80.00 & 3.73 & 0.32 & $>32$ & 0.853 & 0.036 & $<0.001^{* *}$ \\
SOFA & 85.45 & 80.00 & 4.27 & 0.18 & $>7$ & 0.883 & 0.035 & $<0.001^{* *}$ \\
APACHE II & 92.73 & 75.56 & 3.79 & 0.09 & $>16$ & 0.893 & 0.033 & $<0.001^{* *}$ \\
\hline
\end{tabular}

The ability of the scoring system to discriminate between survivor and non-survivors was assessed using the area under ROC. The ROC curves with CTP, MELD, MELD Na, SOFA and APACHE II scores of each patient as independent variable and mortality as outcome was plotted.

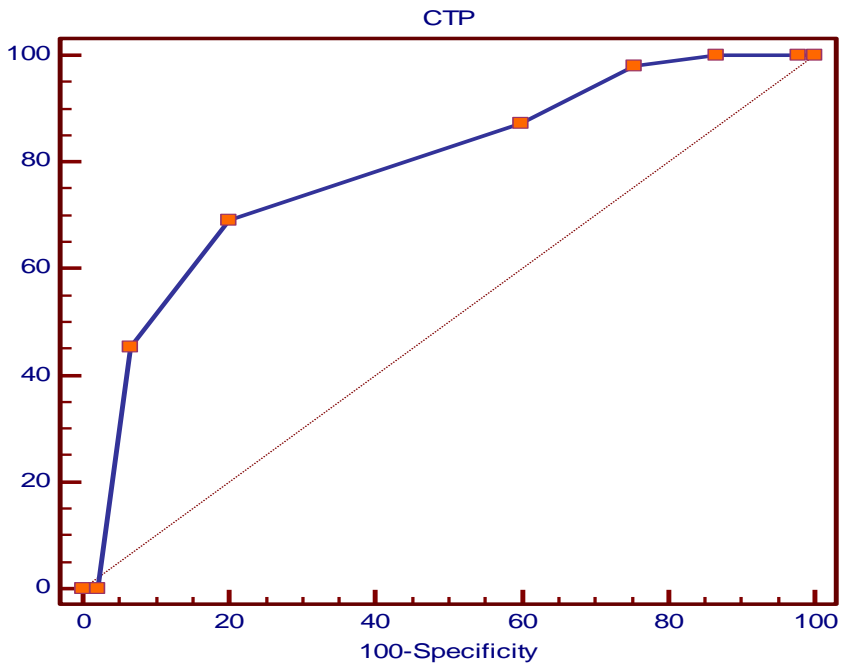



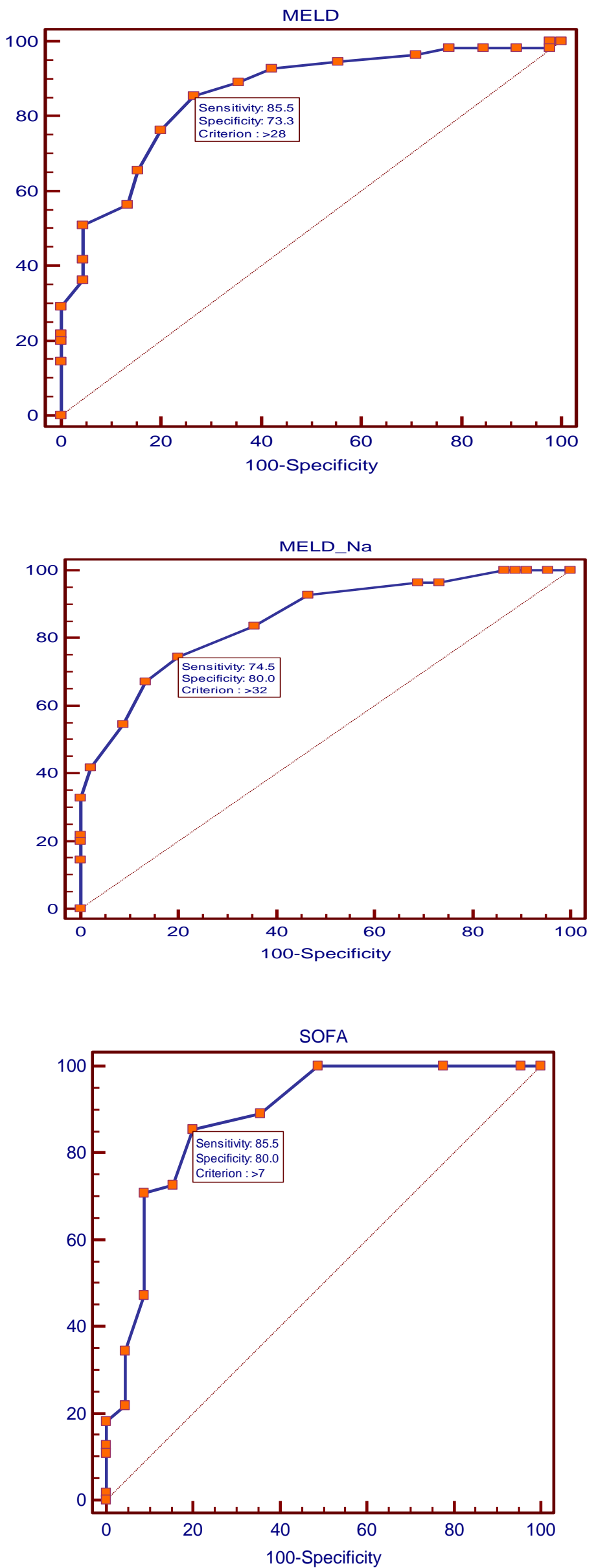


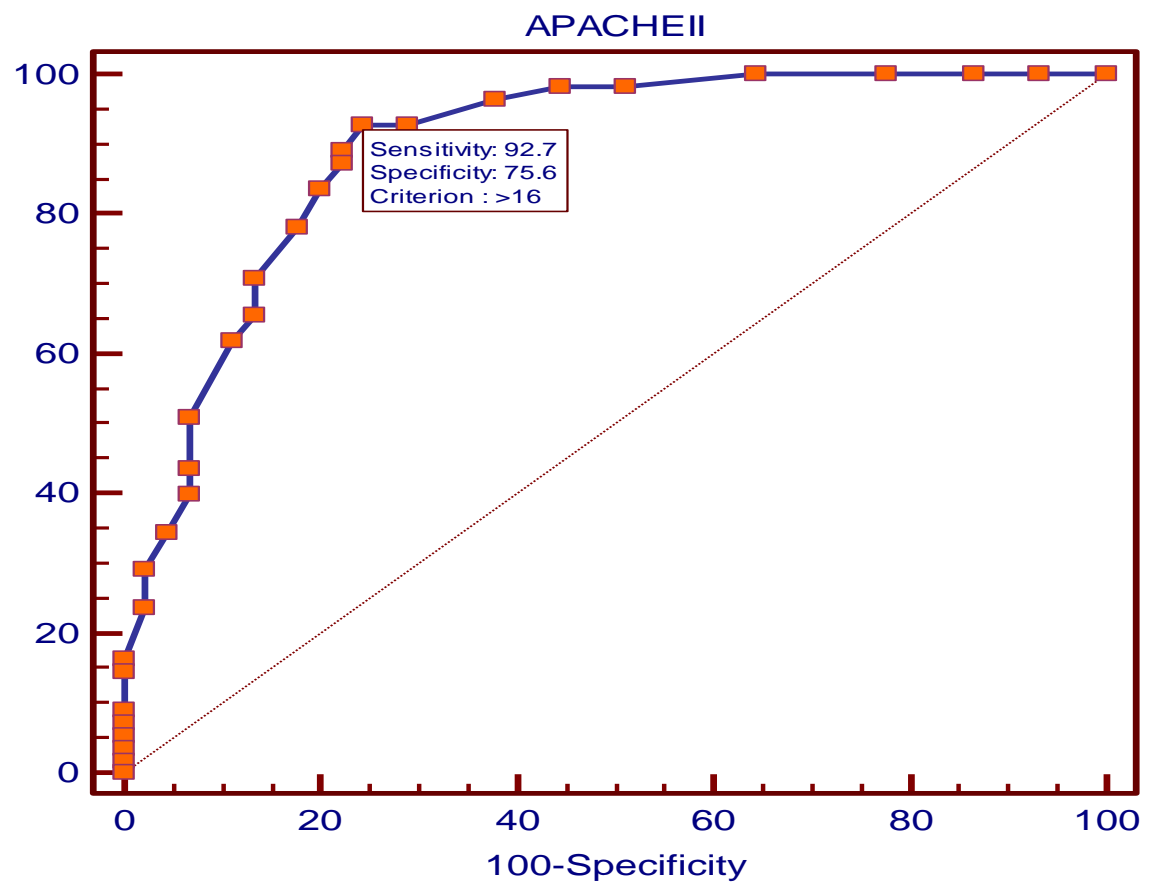

\section{CONCLUSION}

Overall, as expected, the non-survivors had higher CLIF-SOFA and APACHE II scores compared to the survivors. In addition, CLIF-SOFA and APACHE II score was superior to MELD, MELD Na, CTP, SOFA score. This data reiterates the importance of organ failures in determining the prognosis. Our study was limited by a small number of enrolled patients and short study duration of 28 day.

\section{REFERENCES}

1. Jalan R, Williams R. Acute-on-chronic liver failure: pathophysiological basis of therapeutic options. Blood Purif 2002;20: 252-261.

2. Jalan R, Stadlbauer V, Sen S, Cheshire L, Chang YM, Mookerjee RP. Role of predisposition, injury, response and organ failure in the prognosis of patients with acute-on-chronic liver failure: a prospective cohort study. Crit Care. 2012 Nov 27;16(6): R227.

3. Sarin SK, Choudhury A, Sharma MK, et al. Acute-on-chronic liver failure: consensus recommendations of the Asian Pacific association for the study of the liver (APASL): an update. Hepatol Int. $2019 \mathrm{Jul}$;13(4):353-390.

4. Sarin SK, Kumar A, Almeida JA, et al. Acute-on-chronic liver failure: consensus recommendations of the Asian Pacific Association for the study of the liver (APASL). Hepatol Int 2009; 3: 269-282.

5. Moreau R, Gines P, Jalan R, et al. Diagnosis, prevalence, and prognosis of acute-onchronic liver failure (ACLF): results of the EASL- chronic liver failure (CLIF) consortium canonic study. J Hepatol 2012; 56: S552-53. 
6. Garg H, Kumar A, Garg V, et al. Clinical profile and predictors of mortality in patients of acute-on-chronic liver failure. Dia Liver Dis 2012; 44: 166-71.

7. Krishna YR, Saraswat VA, Das K, et al. Clinical features and predictors of outcome in acute hepatitis A and hepatitis E virus hepatitis on cirrhosis. Liver Int 2009;29: 392-8.

8. Zheng MH, Shi KQ, Fan YC, et al. A model to determine 3-month mortality risk in patients with acute on chronic hepatitis B liver failure. Clin Gastroenterol Hepatol 2011;9: 351-6.

9. Vincent J, Moreno R, Takala J, et al. The SOFA (Sepsis related Organ Failure Assessment) score to describe organ dysfunction/failure. Intesive Care Med. 1996; 22:707-10.

10. Kumar Acharya S, Kumar Sharma P, Singh R, et al. Hepatitis E virus (HEV) infection in patients with cirrhosis is associated with rapid decompensation and death. J Hepatol 2007;46: 387-394.

11. Duseja A, Chawla YK, Dhiman RK, Kumar A, Choudhary N, Taneja S. Non-hepatic insults are common acute precipitants in patients with acute on chronic liver failure (ACLF). Dig Dis Sci 2010; 55: 3188-3192.

12. Moreau R, Jalan R, Gines P, Pavesi M, Angeli P, Cordoba J, et al.; Acute-on-chronic liver failure is a distinct syndrome that develops in patients with acute decompensation of cirrhosis. Gastroenterology. 2013 Jun;144(7):1426-37, 1437.e1-9.

13. Amarapurkar D, Dharod MV, Chandnani M, Baijal R, Kumar P, Jain M, Patel N, Kamani P, Issar S, Shah N, Kulkarni S, Gautam S, Shah A, Doshi S. Acute-on-chronic liver failure: a prospective study to determine the clinical profile, outcome, and factors predicting mortality. Indian J Gastroenterol 2015;34: 216-224.

14. Xia Q, Dai X, Zhang Y, Guo Y, Xu X, Yang Q, Du W, Liu X, Chen Y, Huang J, Li L. A modified MELD model for Chinese pre-ACLF and ACLF patients and it reveals poor prognosis in pre-ACLF patients. PLoS One 2013;8: e64379.

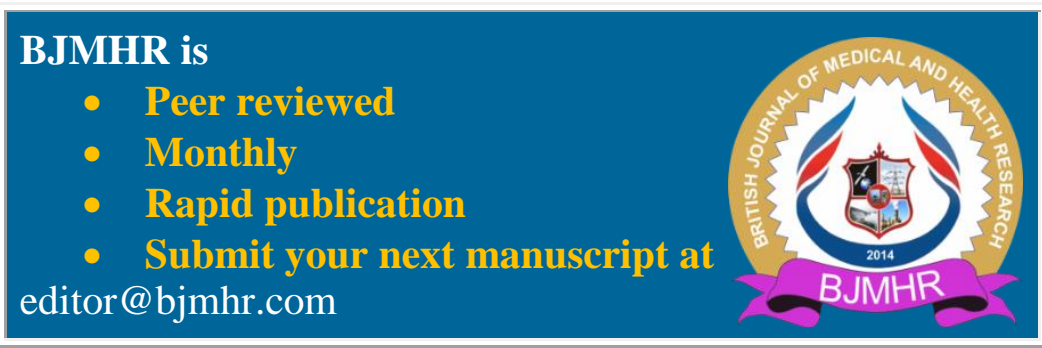

